\title{
The generalized Heisenberg group arising from Weyl relations
}

\author{
Habib Rebei
}

Received: 18 April 2015 / Accepted: 31 July 2015 / Published online: 14 August 2015

(C) Chapman University 2015

\begin{abstract}
In this paper, we introduce the generalized Weyl operators canonically associated with the one-mode oscillator Lie algebra as unitary operators acting on the bosonic Fock space $\Gamma(\mathbb{C})$. Next, we establish the generalized Weyl relations and deduce a group structure on the manifold $\mathbb{R}^{2} \times[-\pi, \pi[\times \mathbb{R}$ generalizing the well-known Heisenberg one in a natural way.
\end{abstract}

Keywords Generalized Weyl operator · Generalized Heisenberg group · Oscillator algebra

Mathematics Subject Classification Primary 81R05; Secondary 22E70 • 20C35

\section{Introduction}

This work is a contribution to the program of the $C^{*}$-algebra second quantization or equivalently, in the case of the oscillator algebra, to give meaning to the formal expressions

$\mathrm{e}^{i\left(a^{+}(\varphi)+a^{-}(\varphi)+n(\phi)\right)} ; \quad \varphi, \phi \in L^{1}(\mathbb{R}) \cap L^{\infty}(\mathbb{R})$

away from the Fock representation. This program was motivated by the construction of the theory of renormalized higher powers of quantum white noise (RHPWN) or equivalently of non-relativistic free Boson fields, see [2] and [3] for more details.

Among the steps of this program is the construction of the Lie group associated with the one-mode version of such given sub-algebra of the RHPWN-algebra. In our case, the group associated to the oscillator algebra is the generalized Heisenberg group. This group will be useful to construct an inductive system of $C^{*}$-algebras each of which will be isomorphic to a finite tensor product of copies of the one-mode algebra, see [1] for more details. Then the program studies the existence of such factorizable state on this system.

Special thanks to Qassim University and its Deanship of Scientific Research for their support to accomplish this work.

H. Rebei $(\varangle)$

Department of Mathematics, College of Science, Qassim University, P.O.Box 5155, Buraidah 51422, Kingdom of Saudi Arabia e-mail: habib.rebei@ipein.rnu.tn 
In this paper, we restrict ourselves to the construction procedure of the generalized Heisenberg group and no approach of this program will be discussed here.

To this goal, we begin by giving the following background:

The one-mode Heisenberg algebra heis(1) is the $*$-Lie algebra generated by $a^{+}, a^{-}$and a central self-adjoint element $1_{0}$ with respect to the relations

$\left[a^{-}, a^{+}\right]=1_{0}, \quad\left(a^{+}\right)^{*}=a^{-}$.

A Fock representation of heis(1) is obtained by giving action of generators $a^{+}, a^{-}$and $1_{0}$ on $\Gamma(\mathbb{C})$ as follows:

$a^{+} \Phi_{n}=\sqrt{n+1} \Phi_{n+1}, \quad n \in \mathbb{N}$,

$a^{-} \Phi_{n}=\sqrt{n} \Phi_{n-1}, \quad n \in \mathbb{N}^{*}:=\mathbb{N} \backslash\{0\}$,

$a^{-} \Phi_{0}=0$

where $\left\{\Phi_{n}, n \in \mathbb{N}\right\}$ is an orthonormal basis of $\Gamma(\mathbb{C})$. Note that for $n \geq 1, \Phi_{n}$ is called the $n$th particle vector and for $n=0, \Phi:=\Phi_{0}$ is called the vacuum vector.

The Weyl operator is defined by

$W(z):=\mathrm{e}^{i\left(z a^{+}+\bar{z} a^{-}\right)} ; \quad z:=x+i y \in \mathbb{C} \equiv \mathbb{R}^{2}$.

Then the Weyl relations are expressed as follows:

$W(z) W\left(z^{\prime}\right)=\mathrm{e}^{i \mathfrak{I m}\left(z \overline{z^{\prime}}\right)} W\left(z+z^{\prime}\right), \quad \Im \mathfrak{I m}\left(z \overline{z^{\prime}}\right)=y x^{\prime}-x y^{\prime}$.

It is well known that if we define $W(z, t)$ by

$\mathbb{C} \times \mathbb{R} \ni(z, t) \mapsto W(z, t):=W(z) \mathrm{e}^{i t}$,

then using (5), we obtain

$W(z, t) W\left(z^{\prime}, t^{\prime}\right)=W\left((z, t) *\left(z^{\prime}, t^{\prime}\right)\right)$,

where $(z, t) *\left(z^{\prime}, t^{\prime}\right)=\left(z+z^{\prime}, t+t^{\prime}+\mathfrak{I m}\left(z \overline{z^{\prime}}\right)\right)$.

So $\{W(z, t), t \in \mathbb{R}, z=x+i y \in \mathbb{C}\}$ is a group and this induces a group law on the manifold $\mathbb{R}^{3} \equiv \mathbb{C} \times \mathbb{R}$ given by:

$(x, y, t) *\left(x^{\prime}, y^{\prime}, t^{\prime}\right)=\left(x+x^{\prime}, y+y^{\prime}, t+t^{\prime}+y x^{\prime}-x y^{\prime}\right)$.

The group Heis $(1):=\left(\mathbb{R}^{3}, *\right)$ is a three-dimensional Lie group called the one-mode Heisenberg group and the map given in (6) is an unitary representation of this group on the Fock space $\Gamma(\mathbb{C})$.

In this paper, the Heisenberg algebra heis(1) will be replaced by the one-mode oscillator algebra $\mathcal{L}_{\text {osc }}(1)$ which is a four-dimensional $*$-Lie algebra with generators $a^{+}, a^{-}, N:=a^{+} a^{-}$and central self-adjoint element $1_{0}$ with the relations

$\left(a^{+}\right)^{*}=a^{-}, \quad\left[a^{-}, a^{+}\right]=1_{0}, \quad\left[N, a^{ \pm}\right]= \pm a^{ \pm}$.

Then, we will consider the representation given from (1) to (3) with additional relation

$N \Phi_{n}=n \Phi_{n}, \quad n \in \mathbb{N}$ 
and we construct the so-called generalized Weyl operator

$W(z, \lambda):=\mathrm{e}^{i\left(z a^{+}+\bar{z} a^{-}+\lambda N\right)}, \quad z \in \mathbb{C}, \lambda \in \mathbb{R}$.

By introducing the rescaled Weyl operator

$W_{r}(z, \lambda, \alpha):=\mathrm{e}^{i \alpha} W(z, \lambda)$,

we will prove a group property

$W_{r}\left(z_{1}, \lambda_{1}, \alpha_{1}\right) W_{r}\left(z_{2}, \lambda_{2}, \alpha_{2}\right)=W_{r}\left(\left(z_{1}, \lambda_{1}, \alpha_{1}\right) \circ\left(z_{2}, \lambda_{2}, \alpha_{2}\right)\right)$,

which induces a group structure on the set $\mathfrak{G}=\mathbb{R}^{2} \times[-\pi, \pi[\times \mathbb{R}$, with law $\circ$ given as in Theorem 3 below.

The manifold with boundary $\mathfrak{G}$ equipped with the law $\circ$ is a Lie group generalizing the well-known Heisenberg group Heis(1), so we will call it the generalized Heisenberg group.

\section{Exponential of generators and exponential Heisenberg group}

This section presents some preliminary results to define the generalized Weyl operator and to write the associated Weyl relations. To this goal, we introduce the exponential vectors given by

$\psi_{x}:=\sum_{n=0}^{+\infty} \frac{x^{n}}{\sqrt{n !}} \Phi_{n} \in \Gamma(\mathbb{C}), \quad x \in \mathbb{C}$.

It is well known that the set of the exponential vectors is total in $\Gamma(\mathbb{C})$. The linear subspace of $\Gamma(\mathbb{C})$ generated by the set of all exponential vectors is called the domain of the exponential vectors. We denote it by $\mathcal{E}$. Moreover, $a^{+}, a^{-}, N$ contain exponential vectors in their domains and actions of these operators are given by the following:

$$
\begin{aligned}
a^{+} \psi_{x} & =\sum_{n=1}^{+\infty} \frac{n x^{n-1}}{\sqrt{n !}} \Phi_{n}, \\
a^{-} \psi_{x} & =x \psi_{x}, \\
N \psi_{x} & =\sum_{n=1}^{+\infty} \frac{n x^{n}}{\sqrt{n !}} \Phi_{n},
\end{aligned}
$$

where the above series converge in $\Gamma(\mathbb{C})$.

Next, we show that the exponential vectors are analytic for $a^{+}, a^{-}$and $N$ so that the operators

$\mathrm{e}^{u a^{+}}, \quad \mathrm{e}^{v N}, \quad \mathrm{e}^{w a^{-}}$

are well defined on the domain $\mathcal{E}$ for all complex numbers $u, v$ and $w$.

Lemma 1 For all $x \in \mathbb{C}$ and $k \in \mathbb{N}^{*}$, the exponential vectors $\psi_{x}$ are in the domain of the operators $\left(a^{+}\right)^{k},\left(a^{-}\right)^{k}$ and $N^{k}$. Moreover, we have

$\left(a^{+}\right)^{k} \psi_{x}=\sum_{n=0}^{+\infty} \frac{\sqrt{(n+k) !}}{n !} x^{n} \Phi_{n+k}$,
$\left(a^{-}\right)^{k} \psi_{x}=x^{k} \psi_{x}$, 


$$
N^{k} \psi_{x}=\sum_{n=0}^{+\infty} \frac{n^{k} x^{n}}{\sqrt{n !}} \Phi_{n} .
$$

Proof From (1), one has

$$
\left(a^{+}\right)^{k} \Phi_{n}=\left(\frac{(n+k) !}{n !}\right)^{\frac{1}{2}} \Phi_{n+k} .
$$

Therefore,

$$
\begin{aligned}
\sum_{n=0}^{+\infty}\left\|\frac{x^{n}}{\sqrt{n !}}\left(a^{+}\right)^{k} \Phi_{n}\right\|^{2} & =\sum_{n=0}^{+\infty}\left|\frac{x^{n}}{\sqrt{n !}}\right|^{2}\left\|\left(\frac{(n+k) !}{n !}\right)^{\frac{1}{2}} \Phi_{n+k}\right\|^{2} \\
& =k ! \sum_{n=0}^{+\infty} \frac{|x|^{2 n}}{n !}\left(\begin{array}{c}
n+k \\
n
\end{array}\right) \\
& \leq k ! \sum_{n=0}^{+\infty} \frac{|x|^{2 n}}{n !} 2^{n+k} \\
& =2^{k} k ! \sum_{n=0}^{+\infty} \frac{\left(2|x|^{2}\right)^{n}}{n !} \\
& =2^{k} k ! \mathrm{e}^{2|x|^{2}} .
\end{aligned}
$$

This gives that $\psi_{x} \in \operatorname{Dom}\left(\left(a^{+}\right)^{k}\right)$.

Using (18), one obtains (15). Moreover, we have

$\left\|\left(a^{+}\right)^{k} \psi_{x}\right\|^{2} \leq 2^{k} k ! \mathrm{e}^{2|x|^{2}}$.

From (13), we deduce that $\psi_{x} \in \operatorname{Dom}\left(\left(a^{-}\right)^{k}\right)$. Then (16) holds.

Using the Eq. (10), we get

$N^{k} \Phi_{n}=n^{k} \Phi_{n}$

Then

$\sum_{n=0}^{+\infty}\left\|\frac{x^{n}}{\sqrt{n !}} N^{k} \Phi_{n}\right\|^{2}=\sum_{n=0}^{+\infty} \frac{|x|^{2 n}}{n !} n^{2 k}$

which converges for all $x \in \mathbb{C}$. This gives that $\psi_{x} \in \operatorname{Dom}\left(N^{k}\right)$. Moreover, we have

$\left\|N^{k} \psi_{x}\right\|^{2}=\sum_{n=0}^{+\infty} n^{2 k} \frac{|x|^{2 n}}{n !}$.

Finally, Eq. (17) is easily obtained by using (20).

Proposition 1 The exponential operators $\mathrm{e}^{u a^{+}}, \mathrm{e}^{v N}$ and $\mathrm{e}^{w a^{-}}$are well defined on the domain of exponential vectors for all complex numbers $u, v$ and $w$. Moreover, the domain $\mathcal{E}$ is invariant under the action of these operators and we have

$\mathrm{e}^{u a^{+}} \psi_{x}=\psi_{x+u}, \quad \mathrm{e}^{v N} \psi_{x}=\psi_{\mathrm{e}^{v} x}, \quad \mathrm{e}^{w a^{-}} \psi_{x}=\mathrm{e}^{w x} \psi_{x}$, 
where $\psi_{x}$ is the exponential vector defined by (11).

Proof To show that the exponential vectors $\psi_{x}$ 's are in the domain of the operators $\mathrm{e}^{u a^{+}}, \mathrm{e}^{w a^{-}}$and $\mathrm{e}^{v N}$, it is sufficient to prove the convergence of the series

(i) $\sum_{k=0}^{+\infty}\left\|\frac{u^{k}}{k !}\left(a^{+}\right)^{k} \psi_{x}\right\|$, (ii) $\sum_{k=0}^{+\infty}\left\|\frac{w^{k}}{k !}\left(a^{-}\right)^{k} \psi_{x}\right\|$ and (iii) $\sum_{k=0}^{+\infty}\left\|\frac{v^{k}}{k !} N^{k} \psi_{x}\right\|$.

Using (19), we deduce that

$\left\|\left(a^{+}\right)^{k} \psi_{x}\right\| \leq 2^{\frac{k}{2}} \sqrt{k !} \mathrm{e}^{|x|^{2}}$.

Then

$$
\begin{aligned}
\sum_{k=0}^{+\infty}\left\|\frac{u^{k}}{k !}\left(a^{+}\right)^{k} \psi_{x}\right\| & \leq \sum_{k=0}^{+\infty} \frac{|u|^{k}}{k !} 2^{\frac{k}{2}} \sqrt{k !} \mathrm{e}^{|x|^{2}} \\
& =\mathrm{e}^{|x|^{2}} \sum_{k=0}^{+\infty} \frac{|\sqrt{2} u|^{k}}{\sqrt{k !}}
\end{aligned}
$$

which converges for all $u \in \mathbb{C}$.

This proves the convergence of (i). Moreover, using (15), one has

$$
\begin{aligned}
\mathrm{e}^{u a^{+}} \psi_{x} & =\sum_{k=0}^{+\infty} \frac{u^{k}}{k !}\left(a^{+}\right)^{k} \psi_{x} \\
& =\sum_{k=0}^{+\infty} \frac{u^{k}}{k !} \sum_{n=0}^{+\infty} \frac{\sqrt{(n+k) !}}{n !} x^{n} \Phi_{n+k} \\
& =\sum_{n, k=0}^{+\infty}\left(\begin{array}{c}
n+k \\
k
\end{array}\right) \frac{u^{k} x^{n}}{\sqrt{(n+k) !}} \Phi_{n+k} \\
& =\sum_{n=0}^{+\infty}\left(\sum_{k=0}^{n}\left(\begin{array}{l}
n \\
k
\end{array}\right) \frac{u^{k} x^{n-k}}{\sqrt{n !}}\right) \Phi_{n} \\
& =\sum_{n=0}^{+\infty} \frac{1}{\sqrt{n !}}\left(\begin{array}{c}
n \\
\sum_{k=0}
\end{array}\left(\begin{array}{l}
n \\
k
\end{array}\right) u^{k} x^{n-k}\right) \Phi_{n} \\
& =\sum_{n=0}^{+\infty} \frac{(x+u)^{n}}{\sqrt{n !}} \Phi_{n} \\
& =\psi_{x+u} .
\end{aligned}
$$

Using (16), we deduce that

$$
\left\|\left(a^{-}\right)^{k} \psi_{x}\right\|=|x|^{k}\left\|\psi_{x}\right\|=|x|^{k} \mathrm{e}^{\frac{|x|^{2}}{2}} .
$$

Then

$$
\begin{aligned}
\sum_{k=0}^{+\infty}\left\|\frac{w^{k}}{k !}\left(a^{-}\right)^{k} \psi_{x}\right\| & =\sum_{k=0}^{+\infty}\left|\frac{w^{k}}{k !}\right||x|^{k} \mathrm{e}^{\frac{|x|^{2}}{2}} \\
& =\mathrm{e}^{\frac{|x|^{2}}{2}} \sum_{k=0}^{+\infty} \frac{|w x|^{k}}{k !} \\
& =\mathrm{e}^{\frac{|x|^{2}}{2}+|w x|} .
\end{aligned}
$$


This proves that the series (ii) converges. Moreover using (16), one obtains

$$
\begin{aligned}
\mathrm{e}^{w a^{-}} \psi_{x} & =\sum_{k=0}^{+\infty} \frac{w^{k}}{k !}\left(a^{-}\right)^{k} \psi_{x} \\
& =\sum_{k=0}^{+\infty} \frac{w^{k}}{k !} x^{k} \psi_{x} \\
& =\sum_{k=0}^{+\infty} \frac{(w x)^{k}}{k !} \psi_{x} \\
& =\mathrm{e}^{w x} \psi_{x} .
\end{aligned}
$$

For all positive sequence $\left(a_{n}\right)$, we have

$$
\sum_{n=0}^{+\infty} a_{n}^{2} \leq\left(\sum_{n=0}^{+\infty} a_{n}\right)^{2}
$$

whenever the series converge. Then, taking $a_{n}=n^{k} \frac{|x|^{n}}{\sqrt{n !}}$ and using (21), we obtain

$\left\|N^{k} \psi_{x}\right\|^{2}=\sum_{n=0}^{+\infty} n^{2 k} \frac{|x|^{2 n}}{n !} \leq\left(\sum_{n=0}^{+\infty} n^{k} \frac{|x|^{n}}{\sqrt{n !}}\right)^{2}$.

This gives

$$
\begin{aligned}
\sum_{k=0}^{+\infty}\left\|\frac{v^{k}}{k !} N^{k} \psi_{x}\right\| & \leq \sum_{k=0}^{+\infty} \frac{|v|^{k}}{k !} \sum_{n=0}^{+\infty} \frac{n^{k}|x|^{n}}{\sqrt{n !}} \\
& =\sum_{n=0}^{+\infty} \frac{|x|^{n}}{\sqrt{n !}} \sum_{k=0}^{+\infty} \frac{n^{k}|v|^{k}}{k !} \\
& =\sum_{n=0}^{+\infty} \frac{|x|^{n}}{\sqrt{n !}} \mathrm{e}^{n|v|} \\
& =\sum_{n=0}^{+\infty} \frac{\left(|x| \mathrm{e}^{|v|}\right)^{n}}{\sqrt{n !}}
\end{aligned}
$$

which converges.

This proves that the series (iii) converges. Moreover using (17), one obtains

$$
\begin{aligned}
\mathrm{e}^{v N} \psi_{x} & =\sum_{k=0}^{+\infty} \frac{v^{k}}{k !} N^{k} \psi_{x} \\
& =\sum_{k=0}^{+\infty} \frac{v^{k}}{k !}\left(\sum_{n=0}^{+\infty} \frac{n^{k} x^{n}}{\sqrt{n !}} \Phi_{n}\right) \\
& =\sum_{n=0}^{+\infty} \frac{x^{n}}{\sqrt{n !}}\left(\sum_{k=0}^{+\infty} \frac{n^{k} v^{k}}{k !}\right) \Phi_{n} \\
& =\sum_{n=0}^{+\infty} \frac{x^{n}}{\sqrt{n !}} \mathrm{e}^{n v} \Phi_{n}
\end{aligned}
$$




$$
\begin{aligned}
& =\sum_{n=0}^{+\infty} \frac{\left(x \mathrm{e}^{v}\right)^{n}}{\sqrt{n !}} \Phi_{n} \\
& =\psi_{\mathrm{e}^{v} x} .
\end{aligned}
$$

To define the generalized Weyl operators, we need to introduce the exponential operators, this is the object of the following definition.

Definition 1 For all complex numbers $u, v, w$ and $\eta$, we define the exponential operator on the domain of the exponential vectors by

$\Gamma(u, v, w, \eta):=\mathrm{e}^{u a^{+}} \mathrm{e}^{v N} \mathrm{e}^{w a^{-}} \mathrm{e}^{\eta}$.

Lemma 2 For all complex numbers $u, v, w$ and $\eta$, the action of the exponential operator $\Gamma(u, v, w, \eta)$ on the domain of exponential vectors $\mathcal{E}$ is given by the following relation

$\Gamma(u, v, w, \eta) \psi_{x}=\mathrm{e}^{\eta+w x} \psi_{\mathrm{e}^{v} x+u}, \quad x \in \mathbb{C}$,

then, we extend (24) by linearity on $\mathcal{E}$. Moreover, we have

$\Gamma\left(u_{1}, v_{1}, w_{1}, \eta_{1}\right) \Gamma\left(u_{2}, v_{2}, w_{2}, \eta_{2}\right)=\Gamma\left(u_{1}+\mathrm{e}^{v_{1}} u_{2}, v_{1}+v_{2}, w_{2}+\mathrm{e}^{v_{2}} w_{1}, \eta_{1}+\eta_{2}+w_{1} u_{2}\right)$,

where $u_{j}, v_{j}, w_{j}, \eta_{j} \in \mathbb{C}, j=1,2$.

Proof Let $u, v, w, \eta, x \in \mathbb{C}$. Then from (22), we obtain for all exponential vector $\psi_{x}$,

$$
\begin{aligned}
\Gamma(u, v, w, \eta) \psi_{x} & =\mathrm{e}^{u a^{+}} \mathrm{e}^{v N} \mathrm{e}^{w a^{-}} \mathrm{e}^{\eta} \psi_{x} \\
& =\mathrm{e}^{\eta+w x} \mathrm{e}^{u a^{+}} \mathrm{e}^{v N} \psi_{x} \\
& =\mathrm{e}^{\eta+w x} \mathrm{e}^{u a^{+}} \psi_{\mathrm{e}^{v} x} \\
& =\mathrm{e}^{\eta+w x} \psi_{\mathrm{e}^{v} x+u} .
\end{aligned}
$$

Now, let $x, u_{j}, v_{j}, w_{j}, \eta_{j} \in \mathbb{C}, j=1,2, \ldots$ Then, using (24), we get for all exponential vector $\psi_{x}$,

$$
\begin{aligned}
& \Gamma\left(u_{1}, v_{1}, w_{1}, \eta_{1}\right) \Gamma\left(u_{2}, v_{2}, w_{2}, \eta_{2}\right) \psi_{x}=\Gamma\left(u_{1}, v_{1}, w_{1}, \eta_{1}\right)\left(\mathrm{e}^{\eta_{2}+w_{2} x} \psi_{\mathrm{e}^{v_{2}} x+u_{2}}\right) \\
& =\mathrm{e}^{\eta_{2}+w_{2} x} \mathrm{e}^{\eta_{1}+w_{1}\left(\mathrm{e}^{v_{2}} x+u_{2}\right)} \psi_{\mathrm{e}^{v_{1}}\left(\mathrm{e}^{v_{2}} x+u_{2}\right)+u_{1}}
\end{aligned}
$$

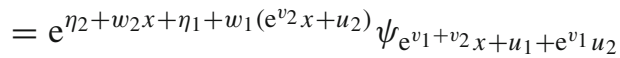

$$
\begin{aligned}
& =\mathrm{e}^{\eta_{1}+\eta_{2}+w_{1} u_{2}+\left(w_{2}+\mathrm{e}^{v_{2}} w_{1}\right) x} \psi_{\mathrm{e}^{v_{1}+v_{2}} x+u_{1}+\mathrm{e}^{v_{1}} u_{2}} \\
& =\Gamma\left(u_{1}+\mathrm{e}^{v_{1}} u_{2}, v_{1}+v_{2}, w_{2}+\mathrm{e}^{v_{2}} w_{1}, \eta_{1}+\eta_{2}+w_{1} u_{2}\right) \psi_{x} .
\end{aligned}
$$

Lemma 3 For all complex numbers $u_{j}, v_{j}, w_{j}, \eta_{j}, j=1,2, \ldots$, we have

$\Gamma\left(u_{1}, v_{1}, w_{1}, \eta_{1}\right)=\Gamma\left(u_{2}, v_{2}, w_{2}, \eta_{2}\right)$,

if and only if,

$u_{1}-u_{2}=w_{1}-w_{2}=0, \quad \eta_{1}-\eta_{2}, v_{1}-v_{2} \in 2 i \pi \mathbb{Z}$. 
Proof From (24), we deduce that

$\Gamma\left(u_{1}, v_{1}, w_{1}, \eta_{1}\right)=\Gamma\left(u_{2}, v_{2}, w_{2}, \eta_{2}\right)$,

if and only if,

$\mathrm{e}^{\eta_{1}+w_{1} x} \psi_{\mathrm{e}^{v_{1}} x+u_{1}}=\mathrm{e}^{\eta_{2}+w_{2} x} \psi_{\mathrm{e}^{v_{2}} x+u_{2}} \forall x \in \mathbb{C}$.

But the set of exponential vectors is linearly independent. Then

- (i) $\mathrm{e}^{v_{1}} x+u_{1}=\mathrm{e}^{v_{2}} x+u_{2} \quad \forall x \in \mathbb{C}$,

- (ii) for all $x \in \mathbb{C}$, there exists $k_{x} \in \mathbb{Z}$ such that

$$
\eta_{1}+w_{1} x=\eta_{2}+w_{2} x+2 i k_{x} \pi .
$$

From (i), we deduce that $u_{1}=u_{2}$ and $\mathrm{e}^{v_{1}}=\mathrm{e}^{v_{2}}$ which gives $v_{1}-v_{2} \in 2 i \pi \mathbb{Z}$.

From (ii), we deduce that $k_{x}$ is continuous in $x$, then it must be constant, (i.e., $k_{x}=k_{0}$ ). It follows that $\left(w_{1}-w_{2}\right) x+\eta_{1}-\eta_{2}-2 i k_{0} \pi=0$ for all $x \in \mathbb{R}$. Then $w_{1}=w_{2}$ and $\eta_{1}-\eta_{2} \in 2 i \pi \mathbb{Z}$.

Remark 1 The above lemma says that

$\Gamma(u, v, w, \eta)=\Gamma\left(u, v+2 i k \pi, w, \eta+2 i k^{\prime} \pi\right) \quad \forall k, k^{\prime} \in \mathbb{Z}$.

Then if the arguments $v$ and $\eta$ are supposed to be in the domain

$D_{\pi}:=\mathbb{R}+i[-\pi, \pi[=\{x+i y ; y \in[-\pi, \pi[\}$,

then the exponential operator $\Gamma(u, v, w, \eta)$ uniquely determines the arguments $u, v, w$ and $\eta$. Hence the set $(\mathbb{C} \times$ $\left.D_{\pi}\right)^{2}$ is the principal domain of the exponential operators

$\{\Gamma(u, v, w, \eta), u, v, w, \eta \in \mathbb{C}\}$.

Easy to prove the following result.

Corollary 1 The set $\mathbb{C}^{4}$ is a group for the law $\star$ given by $\left(u_{1}, v_{1}, w_{1}, \eta_{1}\right) \star\left(u_{2}, v_{2}, w_{2}, \eta_{2}\right)=\left(u_{1}+\mathrm{e}^{v_{1}} u_{2}, v_{1}+v_{2}, w_{2}+\mathrm{e}^{v_{2}} w_{1}, \eta_{1}+\eta_{2}+w_{1} u_{2}\right)$.

Definition 2 The group $\left(\mathbb{C}^{4}, \star\right)$ is called the exponential Heisenberg group. We denote it by Exp-Heis.

\section{The generalized Weyl operator}

Let us consider the following operator-valued function

$V: t \longmapsto V(t):=\Gamma(u(t), v(t), w(t), \eta(t))$,

where $t \longmapsto u(t), v(t), w(t), \eta(t)$ are a continuous $\mathbb{C}$-valued functions on $\mathbb{R}$.

If $(V(t), t \in \mathbb{R})$ is a one-parameter unitary group, then $V(0)=1$. Therefore, according to Lemma 3, we get $u(0)=w(0)=0$ and $v(0), \eta(0) \in 2 i \pi \mathbb{Z}$.

Hence

$\Gamma(u(t), v(t)-v(0), w(t), \eta(t)-\eta(0))=\Gamma(u(t), v(t), w(t), \eta(t))$.

From (27), we can easily see that the study of the operators $\{V(t), t \in \mathbb{R}\}$ with $v(0), \eta(0) \in 2 i \pi \mathbb{Z}$ can be reduced to the study of $\{V(t), t \in \mathbb{R}\}$ with $v(0)=\eta(0)=0$. So, henceforth and without loss of generality, we will suppose that

$v(0)=\eta(0)=0$. 
Proposition 2 Let $u, v, w, \eta$ be the continuous $\mathbb{C}$-valued functions on $\mathbb{R}$ satisfying condition (28). Then the operatorvalued function

$t \longmapsto V(t):=\Gamma(u(t), v(t), w(t), \eta(t))$

is a strongly continuous one-parameter unitary group, if and only if, there exits $(z, \lambda, \alpha) \in \mathbb{C} \times \mathbb{R}^{2}$ such that:

$u(t)=e_{1}(i \lambda t) i z t, \quad v(t)=i \lambda t, \quad w(t)=e_{1}(i \lambda t) i \bar{z} t, \quad \eta(t)=i \alpha t-|z t|^{2} e_{2}(i \lambda t)$,

where the functions $e_{1}$ and $e_{2}$ are given by the following:

$e_{1}(x):=\sum_{n=1}^{+\infty} \frac{x^{n-1}}{n !}=\frac{\mathrm{e}^{x}-1}{x}, \quad x \in \mathbb{C}$,

$e_{2}(x):=\sum_{n=2}^{+\infty} \frac{x^{n-2}}{n !}=\frac{\mathrm{e}^{x}-x-1}{x^{2}}, \quad x \in \mathbb{C}$.

Remark 2 The functions $e_{1}$ and $e_{2}$ satisfy the following relations

$$
\begin{aligned}
\overline{e_{1}(i x)} & =e_{1}(-i x), \quad x \in \mathbb{R} . \\
\mathrm{e}^{-x} e_{1}(x) & =e_{1}(-x) ; \quad e_{2}(-x)+e_{2}(-x)=e_{1}(x) e_{1}(-x), \quad x \in \mathbb{C} .
\end{aligned}
$$

Using Theorem VIII.8 in [4] and Proposition 2, we get the following corollary

Corollary 2 The one-parameter unitary group $\{V(t), t \in \mathbb{R}\}$ takes the form

$V(t)=\mathrm{e}^{i t G}, \quad t \in \mathbb{R}$,

where $G$ is a self-adjoint operator, i.e., $G$ is the Stone generator of $V(t), t \in \mathbb{R}$.

Proof (of Proposition 2).

The group property

$(V(t))^{*}=(V(t))^{-1}=V(-t)$

gives

$\Gamma(\overline{w(t)}, \overline{v(t)}, \overline{u(t)}, \overline{\eta(t)})=\Gamma(u(-t), v(-t), w(-t), \eta(-t))$.

From Lemma 3, we deduce that $\forall t \in \mathbb{R}$,

$\overline{w(t)}=u(-t)$,

$\overline{u(t)}=w(-t)$,

$\overline{v(t)}=v(-t)+2 i k_{t} \pi$

$\overline{\eta(t)}=\eta(-t)+2 i k_{t}^{\prime} \pi$

where $k_{t}, k_{t}^{\prime} \in \mathbb{Z}$.

Note that Eqs. (36) and (35) are equivalent.

Since the functions $v(t)$ and $\eta(t)$ are continuous then it is the same for the $\mathbb{Z}$-valued functions $k_{t}$ and $k_{t}^{\prime}$. This gives $k_{t}=k_{0}$ and $k_{t}^{\prime}=k_{0}^{\prime}$. Taking $t=0$, we obtain $k_{0}^{\prime}=k_{0}=0$.

Equations from (35) to (38) become

$$
\begin{aligned}
& \overline{w(t)}=u(-t), \\
& \overline{v(t)}=v(-t), \\
& \overline{\eta(t)}=\eta(-t) .
\end{aligned}
$$


From Eq. (25), we get

$$
\begin{aligned}
V(t) V(s)= & \Gamma(u(t), v(t), w(t), \eta(t)) \Gamma(u(s), v(s), w(s), \eta(s)) \\
= & \Gamma\left(u(t)+\mathrm{e}^{v(t)} u(s), v(t)+v(s), w(s)+\mathrm{e}^{v(s)} w(t),\right. \\
& \eta(t)+\eta(s)+w(t) u(s)) .
\end{aligned}
$$

On the other hand, we have

$V(t) V(s)=V(t+s)=\Gamma(u(t+s), v(t+s), w(t+s), \eta(t+s))$.

Then, using Lemma 3, we obtain

$$
\begin{aligned}
u(t+s) & =u(t)+\mathrm{e}^{v(t)} u(s), \\
v(s+t) & =v(t)+v(s)+2 i k_{s, t} \pi, \quad k_{s, t} \in \mathbb{Z}, \\
w(s+t) & =w(s)+\mathrm{e}^{v(s)} w(t), \\
\eta(s+t) & =\eta(t)+\eta(s)+w(t) u(s)+2 i k_{s, t}^{\prime} \pi, \quad k_{s, t}^{\prime} \in \mathbb{Z} .
\end{aligned}
$$

Taking $s=t=0$, with continuity of the arguments, one obtains $k_{s, t}=k_{s, t}^{\prime}=0$.

From Eq. (43) and since $v$ is continuous, we obtain

$v(t)=c t, \quad c \in \mathbb{C}$,

and from Eq. (40) the constant $c$ must be purely imaginary, i.e., $c=i \lambda$ for some $\lambda \in \mathbb{R}$.

Note that we deduce from Eqs. (39) and (40) that Eqs. (42) and (44) are equivalent.

In this step, we investigate to resolve Eq. (42) which becomes as follows

$u(s+t)=u(t)+\mathrm{e}^{i \lambda t} u(s)$.

For all $t \in \mathbb{R}, p \in \mathbb{Z}$ such that $\lambda t \notin 2 \pi \mathbb{Z}$, we have

$$
\begin{aligned}
u(p t)= & u((p-1) t)+\mathrm{e}^{i \lambda(p-1) t} u(t) \\
= & u((p-2) t)+\mathrm{e}^{i \lambda(p-2) t} u(t)+\mathrm{e}^{i \lambda(p-1) t} u(t) \\
& \vdots \\
= & u(t) \sum_{k=0}^{p-1} \mathrm{e}^{i \lambda k t} \\
= & u(t) \frac{\mathrm{e}^{i \lambda p t}-1}{\mathrm{e}^{i \lambda t}-1} .
\end{aligned}
$$

Taking $t=\frac{1}{p}$ in Eq. (47) and replacing $p$ by $q$, we get

$$
u(1)=u\left(\frac{1}{q}\right) \frac{\mathrm{e}^{i \lambda}-1}{\mathrm{e}^{i \frac{\lambda}{q}}-1} .
$$

Taking $t=\frac{1}{q}$ in Eq. (47), one obtains

$$
u\left(\frac{p}{q}\right)=u\left(\frac{1}{q}\right) \frac{\mathrm{e}^{i \lambda \frac{p}{q}}-1}{\mathrm{e}^{i \frac{\lambda}{q}}-1} .
$$

Combining Eqs. (48) and (49), one deduces

$$
u\left(\frac{p}{q}\right)=u(1) \frac{\mathrm{e}^{i \lambda \frac{p}{q}}-1}{\mathrm{e}^{i \lambda}-1} \text {. }
$$


Then

$u(t)=u(1) \frac{\mathrm{e}^{i \lambda t}-1}{\mathrm{e}^{i \lambda}-1} \quad \forall t \in \mathbb{Q}$.

Using density of $\mathbb{Q}$ in $\mathbb{R}$ and continuity of $u$, we deduce that the expression (50) holds also for all $t \in \mathbb{R}$. Taking $z=\frac{\lambda u(1)}{\mathrm{e}^{i \lambda}-1}$ and using (39), we obtain the expressions of $u$ and $w$ in (29).

From (45) and using expressions of $u$ and $w$ in (29), we obtain

$$
\begin{aligned}
\eta(t+s)= & \eta(t)+\eta(s)+\frac{|z|^{2}}{\lambda^{2}}\left(\mathrm{e}^{i \lambda s}-1\right)\left(\mathrm{e}^{i \lambda t}-1\right) \\
= & \eta(t)+\eta(s)+\frac{|z|^{2}}{\lambda^{2}}\left(\left(\mathrm{e}^{i \lambda(t+s)}-1\right)-\left(\mathrm{e}^{i \lambda t}-1\right)-\left(\mathrm{e}^{i \lambda s}-1\right)\right) \\
= & \eta(t)-\frac{|z|^{2}}{\lambda^{2}}\left(\mathrm{e}^{i \lambda t}-1\right)+\eta(s)-\frac{|z|^{2}}{\lambda^{2}}\left(\mathrm{e}^{i \lambda s}-1\right) \\
& +\frac{|z|^{2}}{\lambda^{2}}\left(\mathrm{e}^{i \lambda(s+t)}-1\right) .
\end{aligned}
$$

If we consider $g(t)=\eta(t)-\frac{|z|^{2}}{\lambda^{2}}\left(\mathrm{e}^{i \lambda t}-1\right)$, one has

$g(t+s)=g(t)+g(s) \quad \forall s, t \in \mathbb{R}$.

Since $g$ is a continuous $\mathbb{C}$-valued function, then there exists $\delta \in \mathbb{C}$ such that

$g(t)=\delta t$.

Then

$\eta(t)=\delta t+\frac{|z|^{2}}{\lambda^{2}}\left(\mathrm{e}^{i \lambda t}-1\right)$.

Using (41), we deduce that $\delta=i \beta$ for some $\beta \in \mathbb{R}$. This gives

$$
\begin{aligned}
\eta(t) & =i \beta t+\frac{|z|^{2}}{\lambda^{2}}\left(\mathrm{e}^{i \lambda t}-1\right) \\
& =i\left(\beta+\frac{|z|^{2}}{\lambda}\right) t+\frac{|z|^{2}}{\lambda^{2}}\left(\mathrm{e}^{i \lambda t}-i \lambda t-1\right) \\
& =i\left(\beta+\frac{|z|^{2}}{\lambda}\right) t-|z t|^{2} \frac{\mathrm{e}^{i \lambda t}-i \lambda t-1}{(i \lambda t)^{2}} \\
& =i\left(\beta+\frac{|z|^{2}}{\lambda}\right) t-|z t|^{2} e_{2}(i \lambda t) \\
& =i \alpha t-|z t|^{2} e_{2}(i \lambda t),
\end{aligned}
$$

where $\alpha=\beta+\frac{|z|^{2}}{\lambda} \in \mathbb{R}$.

Conversely if $u, v, w, \eta$ are given as in (29), then a straightforward computation gives

$V(t+s)=V(t) V(s), \quad(V(t))^{*} V(t)=1$.

This gives that $V$ is a one-parameter unitary group. Moreover,

$$
\begin{aligned}
\left\|V(t) \psi_{x}-\psi_{x}\right\|^{2} & =\left\|V(t) \psi_{x}\right\|^{2}+\left\|\psi_{x}\right\|^{2}-2 \mathfrak{R e}\left(\left\langle\psi_{x}, V(t) \psi_{x}\right\rangle\right) \\
& =2\left\|\psi_{x}\right\|^{2}-2 \mathfrak{R e}\left(\left\langle\psi_{x}, \mathrm{e}^{w(t) x+\eta(t)} \psi_{\mathrm{e}^{v(t)} x+u(t)}\right\rangle\right) \\
& =2 \mathrm{e}^{|x|^{2}}-2 \mathfrak{R e}\left(\mathrm{e}^{w(t) x+\eta(t)+\bar{x}\left(\mathrm{e}^{v(t)} x+u(t)\right)}\right) .
\end{aligned}
$$


Then

$$
\begin{aligned}
\lim _{t \rightarrow 0}\left\|V(t) \psi_{x}-\psi_{x}\right\|^{2} & =2 \mathrm{e}^{|x|^{2}}-2 \mathfrak{R e}\left(\mathrm{e}^{w(0) x+\eta(0)+\bar{x}\left(\mathrm{e}^{v(0)} x+u(0)\right)}\right) \\
& =2 \mathrm{e}^{|x|^{2}}-2 \mathrm{e}^{|x|^{2}} \\
& =0 .
\end{aligned}
$$

Hence for all exponential vector $\psi=\psi_{x}$, we have

$\lim _{t \rightarrow 0}\|V(t) \psi-\psi\|^{2}=0$.

The above equation can be extended by linearity to the space $\mathcal{E}$.

We will extend $(51)$ on $\Gamma(\mathbb{C})$. Let $\Gamma(\mathbb{C}) \ni \psi:=\lim _{n \rightarrow+\infty} \psi_{n}, \psi_{n} \in \mathcal{E}$; then we have

$$
\begin{aligned}
\|V(t) \psi-\psi\|^{2} & =\left\|\left(V(t) \psi-V(t) \psi_{n}\right)+\left(V(t) \psi_{n}-\psi_{n}\right)+\left(\psi_{n}-\psi\right)\right\|^{2} \\
& \leq\left\|V(t)\left(\psi-\psi_{n}\right)\right\|^{2}+\left\|V(t) \psi_{n}-\psi_{n}\right\|^{2}+\left\|\psi_{n}-\psi\right\|^{2} \\
& =2\left\|\psi_{n}-\psi\right\|^{2}+\left\|V(t) \psi_{n}-\psi_{n}\right\|^{2} .
\end{aligned}
$$

While $\psi=\lim _{n \rightarrow+\infty} \psi_{n}$, then for all $\epsilon>0$, there exits $n_{0} \in \mathbb{N}$ such that,

$\left\|\psi_{n}-\psi\right\|^{2} \leq \frac{\epsilon}{4} \quad \forall n \geq n_{0}$.

From Eq. (51), there exists $\kappa>0$, such that

$\left\|V(t) \psi_{n_{0}}-\psi_{n_{0}}\right\|^{2} \leq \frac{\epsilon}{2} \quad \forall|t| \leq \kappa$.

Combining the above equations, one obtains

$\|V(t) \psi-\psi\|^{2} \leq 2\left\|\psi_{n_{0}}-\psi\right\|^{2}+\left\|V(t) \psi_{n_{0}}-\psi_{n_{0}}\right\|^{2} \leq 2 \frac{\epsilon}{4}+\frac{\epsilon}{2}=\epsilon \quad \forall|t| \leq \kappa$.

Hence $V$ is strongly continuous.

Theorem 1 Let $u(t), v(t), w(t), \eta(t)$ be functions given as in $E q$. (29) such that $\alpha=0$. Then the Stone generator of $V(t)$ and the field operator

$H(z, \lambda):=z a^{+}+\bar{z} a^{-}+\lambda N$

coincide on the domain $\mathcal{E}$ of the exponential vectors, i.e.,

$V(t)=\mathrm{e}^{i t\left(z a^{+}+\bar{z} a^{-}+\lambda N\right)}, \quad t \in \mathbb{R}$,

on $\mathcal{E}$.

Definition 3 The unitary operator

$W(z, \lambda):=\mathrm{e}^{z a^{+}+\bar{z} a^{-}+\lambda N}$

is called the generalized Weyl operator.

Proof (of Theorem 1).

We have

$V(t)=\mathrm{e}^{i t G}, t \in \mathbb{R}$. 
Then, by taking the derivative of (53), we get

$$
\begin{aligned}
&\left\langle\psi_{y}, i G \psi_{x}\right\rangle=\left\langle\psi_{y}, \frac{\mathrm{d}}{\mathrm{d} t} \mid t=0 V(t) \psi_{x}\right\rangle \\
&=\frac{\mathrm{d}}{\mathrm{d} t} \mid t=0 \\
&=\frac{\mathrm{d}}{\mathrm{d} t} \mid t=0 \\
&\left.=\frac{\mathrm{d}}{\mathrm{d} t}\left|t=0, V(t) \psi_{x}\right\rangle, \mathrm{e}^{\eta(t)+w(t) x} \psi_{\mathrm{e}^{v(t)} x+u(t)}\right\rangle \\
&=\frac{\mathrm{d}}{\mathrm{d} t} \mid t=0 \\
& \mathrm{e}^{\eta(t)+w(t) x+\bar{y}\left(\mathrm{e}^{v(t)} x+u(t)\right)} \mathrm{e}^{\bar{y}\left(\mathrm{e}^{v(t)} x+u(t)\right)} \\
&=\frac{\mathrm{d}}{\mathrm{d} t} \mid t=0 \\
& \mathrm{e}^{h(t)} \\
&=h^{\prime}(0) \mathrm{e}^{h(0)},
\end{aligned}
$$

where

$h(t):=\eta(t)+w(t) x+\bar{y}\left(\mathrm{e}^{v(t)} x+u(t)\right)$.

Clearly $h(0)=\bar{y} x$ and a straightforward calculation gives

$$
\begin{aligned}
h^{\prime}(0) & =\eta^{\prime}(0)+w^{\prime}(0) x+\bar{y}\left(v^{\prime}(0) \mathrm{e}^{v(0)} x+u^{\prime}(0)\right) \\
& =i \bar{z} x+\bar{y}(i \lambda x+i z) \\
& =i(\bar{z} x+\bar{y} x \lambda+z \bar{y}) .
\end{aligned}
$$

Then

$$
\left\langle\psi_{y}, G \psi_{x}\right\rangle=(\bar{z} x+\bar{y} x \lambda+z \bar{y}) \mathrm{e}^{\bar{y} x} .
$$

On the other hand, we have

$$
\begin{aligned}
& \left\langle\psi_{y}, i H(z, \lambda) \psi_{x}\right\rangle=\left\langle\psi_{y}, i\left(z a^{+}+\bar{z} a^{-}+\lambda N\right) \psi_{x}\right\rangle \\
& =i\left(\left\langle\psi_{y}, z a^{+} \psi_{x}\right\rangle+\left\langle\psi_{y}, \bar{z} a^{-} \psi_{x}\right\rangle+\left\langle\psi_{y}, \lambda N \psi_{x}\right\rangle\right) \\
& =i\left(z\left\langle a^{-} \psi_{y}, \psi_{x}\right\rangle+\bar{z}\left\langle\psi_{y}, a^{-} \psi_{x}\right\rangle+\lambda\left\langle\psi_{y}, N \psi_{x}\right\rangle\right)
\end{aligned}
$$

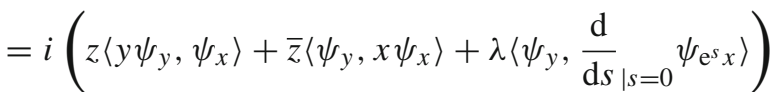

$$
\begin{aligned}
& \left.=i\left(z\left\langle y \psi_{y}, \psi_{x}\right\rangle+\bar{z}\left\langle\psi_{y}, x \psi_{x}\right\rangle+\lambda \frac{\mathrm{d}}{\mathrm{d} s}|s=0| \psi_{y}, \psi_{\mathrm{e}^{s} x}\right\rangle\right) \\
& =i\left(z \bar{y} \mathrm{e}^{\bar{y} x}+\bar{z} x \mathrm{e}^{\bar{y} x}+\lambda \frac{\mathrm{d}}{\mathrm{d} s} \mid s=0 . \mathrm{e}^{\bar{y} \mathrm{e}^{s} x}\right) \\
& =i\left(z \bar{y} \mathrm{e}^{\bar{y} x}+\bar{z} x \mathrm{e}^{\bar{y} x}+\lambda x \bar{y} \mathrm{e}^{\bar{y} x}\right) \\
& =i(z \bar{y}+\bar{z} x+\lambda x \bar{y}) \mathrm{e}^{\bar{y} x} \text {. }
\end{aligned}
$$

Combining (54) and (55), we get

$$
\left\langle\psi_{y},(G-H(z, \lambda)) \psi_{x}\right\rangle=0 \quad \forall x, y \in \mathbb{C} .
$$

Extending the above relation by linearity, we get $G=H(z, \lambda)$ on $\mathcal{E}$. 


\section{The generalized Heisenberg group}

We introduce the rescaled Weyl operator defined by:

$W_{r}(z, \lambda, \alpha):=\mathrm{e}^{i \alpha} W(z, \lambda), \quad(z, \lambda, \alpha) \in \mathbb{C} \times \mathbb{R}^{2}$.

In the following lemma, we will give a suitable domain $\mathfrak{D}$ for which $W_{r}(z, \lambda, \alpha)$ determines uniquely the arguments $z$ and $\lambda$ and $\alpha$.

Lemma 4 Let $\mathfrak{D}=\mathbb{C} \times\left[-\pi, \pi\left[{ }^{2}\right.\right.$. Then for all $(z, \lambda, \alpha),\left(z^{\prime}, \lambda^{\prime}, \alpha^{\prime}\right) \in \mathfrak{D}$, we have

$W_{r}(z, \lambda, \alpha)=W_{r}\left(z^{\prime}, \lambda^{\prime}, \alpha^{\prime}\right)$,

if and only if,

$(z, \lambda, \alpha)=\left(z^{\prime}, \lambda^{\prime}, \alpha^{\prime}\right)$.

Proof We have

$W_{r}(z, \lambda, \alpha)=\Gamma\left(i z e_{1}(i \lambda), i \lambda, i \bar{z} e_{1}(i \lambda), i \alpha-|z|^{2} e_{2}(i \lambda)\right)$

Then from Lemma 3 ,

$W_{r}(z, \lambda, \alpha)=W_{r}\left(z^{\prime}, \lambda^{\prime}, \alpha^{\prime}\right)$,

if and only if,

$$
\begin{aligned}
& i z e_{1}(i \lambda)=i z^{\prime} e_{1}\left(i \lambda^{\prime}\right), \\
& i \bar{z} e_{1}(i \lambda)=i \bar{z}^{\prime} e_{1}\left(i \lambda^{\prime}\right), \\
& i \lambda-i \lambda^{\prime} \in 2 i \pi \mathbb{Z}, \\
& i \alpha-|z|^{2} e_{2}(i \lambda)-\left(i \alpha^{\prime}-\left|z^{\prime}\right|^{2} e_{2}\left(i \lambda^{\prime}\right)\right) \in 2 i \pi \mathbb{Z} .
\end{aligned}
$$

From (59) and using the fact that $\lambda, \lambda^{\prime} \in\left[-\pi, \pi\right.$, we deduce that $\lambda=\lambda^{\prime}$. While for $\lambda \in\left[-\pi, \pi\left[, e_{1}(i \lambda) \neq 0\right.\right.$, then from (57), we obtain $z=z^{\prime}$. Using (60) and the fact that $\alpha, \alpha^{\prime} \in\left[-\pi, \pi\right.$, we deduce that $\alpha=\alpha^{\prime}$.

Theorem 2 For all $\left(z_{j}, \lambda_{j}, \alpha_{j}\right) \in \mathfrak{D}, j=1,2$, there exists a unique $(z, \lambda, \alpha) \in \mathfrak{D}$ satisfying

$W_{r}\left(z_{1}, \lambda_{1}, \alpha_{1}\right) W_{r}\left(z_{2}, \lambda_{2}, \alpha_{2}\right)=W_{r}(z, \lambda, \alpha)$.

Moreover, the triple $(z, \lambda, \alpha)$ is given by:

$\lambda=\lambda_{1}+\lambda_{2} \bmod (2 \pi \mathbb{Z})$,

$z=\frac{e_{1}\left(i \lambda_{1}\right) z_{1}+\mathrm{e}^{i \lambda_{1}} e_{1}\left(i \lambda_{2}\right) z_{2}}{e_{1}(i \lambda)}$

$\alpha=\alpha_{1}+\alpha_{2}+i\left(e_{2}\left(i \lambda_{1}\right)\left|z_{1}\right|^{2}+e_{2}\left(i \lambda_{2}\right)\left|z_{2}\right|^{2}-e_{2}(i \lambda)|z|^{2}+e_{1}\left(i \lambda_{1}\right) e_{1}\left(i \lambda_{2}\right) \overline{z_{1}} z_{2}\right) \bmod (2 \pi \mathbb{Z})$.

Remark 3 In Eq. (62), the constraint for the value of $\lambda$ to be in $[-\pi, \pi$ [ guaranties the existence of the solution $z$ of Eq. (73) below. Hence Eq. (62) makes a sense. In fact, if $\lambda \in 2 \pi \mathbb{Z}$, then $e_{1}(i \lambda)=0$ and Eq. (73) may not have a solution. But with this choice, a solution is sure and unique. Moreover, we have not lost generality. Indeed from Lemma 3, we deduce that

$W(z, \lambda+2 k \pi, \alpha)=W(z, \lambda, \alpha)$.

Thus the Weyl relations can be extended on the domain $\mathbb{C} \times \mathbb{R}^{2}$ by allowing $\lambda_{1}, \lambda_{2} \in \mathbb{R}$, but we take $\lambda \in[-\pi, \pi[$ which is given by Eq. (62). For the arguments $z$ and $\alpha$, we keep the same expressions in (63) and (64) but we remove $(\bmod (2 \pi \mathbb{Z}))$ from $(64)$. Hence the extended Weyl relations will be given by the following equations

$W_{r}\left(z_{1}, \lambda_{1}, \alpha_{1}\right) W_{r}\left(z_{2}, \lambda_{2}, \alpha_{2}\right)=W_{r}(z, \lambda, \alpha), \quad\left(z_{i}, \lambda_{i}, \alpha_{i}\right) \in \mathbb{C} \times \mathbb{R}^{2}$, 
where the triple $(z, \lambda, \alpha) \in \mathbb{C} \times[-\pi, \pi[\times \mathbb{R}$ is given by

$\lambda=\lambda_{1}+\lambda_{2} \bmod (2 \pi \mathbb{Z})$,

$z=\frac{e_{1}\left(i \lambda_{1}\right) z_{1}+\mathrm{e}^{i \lambda_{1}} e_{1}\left(i \lambda_{2}\right) z_{2}}{e_{1}(i \lambda)}$

$\alpha=\alpha_{1}+\alpha_{2}+i\left(e_{2}\left(i \lambda_{1}\right)\left|z_{1}\right|^{2}+e_{2}\left(i \lambda_{2}\right)\left|z_{2}\right|^{2}-e_{2}(i \lambda)|z|^{2}\right.$

$\left.+e_{1}\left(i \lambda_{1}\right) e_{1}\left(i \lambda_{2}\right) \bar{z}_{1} z_{2}\right)$.

Corollary 3 The generalized Weyl relations hold:

$W\left(z_{1}, \lambda_{1}\right) W\left(z_{2}, \lambda_{2}\right)=\mathrm{e}^{i \gamma} W(z, \lambda)$,

where $\lambda \in[-\pi, \pi[$ and $z \in \mathbb{C}$ are given as in (66) and (67) and

$\gamma=i\left(e_{2}\left(i \lambda_{1}\right)\left|z_{1}\right|^{2}+e_{2}\left(i \lambda_{2}\right)\left|z_{2}\right|^{2}-e_{2}(i \lambda)|z|^{2}+e_{1}\left(i \lambda_{1}\right) e_{1}\left(i \lambda_{2}\right) \overline{z_{1}} z_{2}\right)$.

Proof Using Eqs. (56) and (65), one has

$$
\begin{aligned}
W\left(z_{1}, \lambda_{1}\right) W\left(z_{2}, \lambda_{2}\right) & =\mathrm{e}^{-i\left(\alpha_{1}+\alpha_{2}\right)} W_{r}\left(z_{1}, \lambda_{1}, \alpha_{1}\right) W_{r}\left(z_{2}, \lambda_{2}, \alpha_{2}\right) \\
& =\mathrm{e}^{-i\left(\alpha_{1}+\alpha_{2}\right)} W_{r}(z, \lambda, \alpha) \\
& =\mathrm{e}^{i\left(\alpha-\alpha_{1}-\alpha_{2}\right)} W(z, \lambda) \\
& =\mathrm{e}^{i \gamma} W(z, \lambda),
\end{aligned}
$$

where $\lambda$ and $z$ are given as in Eqs. (66) and (67) and

$\gamma=\alpha-\alpha_{1}-\alpha_{2}$.

Using Eq. (68), one obtains (70).

Proof (of Theorem 2).

We will use the notation

$W_{r}(z, \lambda, \alpha)=\Gamma(u, v, w, \eta)$,

where

$u=i z e_{1}(i \lambda) ; \quad w=i \bar{z} e_{1}(i \lambda) ; \quad v=i \lambda ; \quad \eta=i \alpha-|z|^{2} e_{2}(i \lambda)$.

From relation (25), we get

$$
\begin{aligned}
W_{1} W_{2}: & =W_{r}\left(z_{1}, \lambda_{1}, \alpha_{1}\right) W_{r}\left(z_{2}, \lambda_{2}, \alpha_{2}\right) \\
& =\Gamma\left(u_{1}, v_{1}, w_{1}, \eta_{1}\right) \Gamma\left(u_{2}, v_{2}, w_{2}, \eta_{2}\right) \\
& =\Gamma\left(u_{1}+\mathrm{e}^{v_{1}} u_{2}, v_{1}+v_{2}, w_{2}+\mathrm{e}^{v_{2}} w_{1}, \eta_{1}+\eta_{2}+w_{1} u_{2}\right) .
\end{aligned}
$$

We will prove that there exits $(z, \lambda, \alpha) \in \mathbb{C} \times\left[-\pi, \pi\left[^{2}\right.\right.$, such that

$u_{1}+\mathrm{e}^{v_{1}} u_{2}=i z e_{1}(i \lambda) ; \quad v_{1}+v_{2}=i \lambda ; \quad w_{2}+\mathrm{e}^{v_{2}} w_{1}=i \bar{z} e_{1}(i \lambda), \quad \eta_{1}+\eta_{2}+w_{1} u_{2}=i \alpha-|z|^{2} e_{2}(i \lambda)$.

Then using (71), one obtains

$W_{1} W_{2}=\Gamma\left(i z e_{1}(i \lambda), i \lambda, i \bar{z} e_{1}(i \lambda), i \alpha-|z|^{2} e_{2}(i \lambda)\right)=W_{r}(z, \lambda, \alpha)$.

The uniqueness comes from Lemma 4. 
Let $\lambda \in[-\pi, \pi[$ such that $\lambda_{1}+\lambda_{2}=\lambda \bmod (2 \pi \mathbb{Z})$

With this choice of $\lambda, e_{1}(i \lambda) \neq 0$. Then we can define $z \in \mathbb{C}$ as follows:

$e_{1}(i \lambda) i z=u_{1}+\mathrm{e}^{v_{1}} u_{2}=i z_{1} e_{1}\left(i \lambda_{1}\right)+i z_{2} \mathrm{e}^{i \lambda_{1}} e_{1}\left(i \lambda_{2}\right)$.

Then

$z=\frac{z_{1} e_{1}\left(i \lambda_{1}\right)+z_{2} \mathrm{e}^{i \lambda_{1}} e_{1}\left(i \lambda_{2}\right)}{e_{1}(i \lambda)}$

Using the property (32), one obtains

$$
\begin{aligned}
i \bar{z} e_{1}(i \lambda) & =i \overline{z e_{1}(-i \lambda)} \\
& =i \overline{\mathrm{e}^{-i \lambda} z e_{1}(i \lambda)} \\
& =i \mathrm{e}^{i \lambda \overline{z e_{1}(i \lambda)}} \\
& =i \mathrm{e}^{i \lambda} \overline{z_{1} e_{1}\left(i \lambda_{1}\right)+z_{2} \mathrm{e}^{i \lambda_{1}} e_{1}\left(i \lambda_{2}\right)} \\
& =i \mathrm{e}^{i \lambda}\left(\bar{z}_{1} e_{1}\left(-i \lambda_{1}\right)+\bar{z}_{2} \mathrm{e}^{-i \lambda_{1}} e_{1}\left(-i \lambda_{2}\right)\right) \\
& =i \mathrm{e}^{i\left(\lambda_{1}+\lambda_{2}\right)}\left(\bar{z}_{1} e_{1}\left(-i \lambda_{1}\right)+\bar{z}_{2} \mathrm{e}^{-i \lambda_{1}} e_{1}\left(-i \lambda_{2}\right)\right) \\
& =i\left(\bar{z}_{1} \mathrm{e}^{i \lambda_{2}} e_{1}\left(i \lambda_{1}\right)+\bar{z}_{2} e_{1}\left(i \lambda_{2}\right)\right) \\
& =w_{2}+\mathrm{e}^{v_{2}} w_{1} .
\end{aligned}
$$

Moreover, we have

$$
\begin{aligned}
\eta_{1}+\eta_{2}+w_{1} u_{2}= & i \alpha_{1}-\left|z_{1}\right|^{2} e_{2}\left(i \lambda_{1}\right)+i \alpha_{2}-\left|z_{2}\right|^{2} e_{2}\left(i \lambda_{2}\right) \\
& -\bar{z}_{1} z_{2} e_{1}\left(i \lambda_{1}\right) e_{1}\left(i \lambda_{2}\right) \\
= & i\left(\alpha_{1}+\alpha_{2}\right)-\left|z_{1}\right|^{2} e_{2}\left(i \lambda_{1}\right)-\left|z_{2}\right|^{2} e_{2}\left(i \lambda_{2}\right) \\
& -\bar{z}_{1} z_{2} e_{1}\left(i \lambda_{1}\right) e_{1}\left(i \lambda_{2}\right) \\
= & i \alpha-|z|^{2} e_{2}(i \lambda) .
\end{aligned}
$$

We will show that $\alpha$ defined by (74) is real, then we can take $\alpha \in[-\pi, \pi[$ as in (64).

From (64), it is sufficient to show that $\theta$ given by

$\theta=\left|z_{1}\right|^{2} e_{2}\left(i \lambda_{1}\right)+\left|z_{2}\right|^{2} e_{2}\left(i \lambda_{2}\right)-|z|^{2} e_{2}(i \lambda)+\bar{z}_{1} z_{2} e_{1}\left(i \lambda_{1}\right) e_{1}\left(i \lambda_{2}\right)$

is purely imaginary, i.e., $\theta \in i \mathbb{R}$. This is equivalent to see that $\theta+\bar{\theta}=0$.

Using (63) with help of properties (32) and (33), one obtains

$$
\begin{aligned}
\theta+\bar{\theta}= & \left|z_{1}\right|^{2} e_{2}\left(i \lambda_{1}\right)+\left|z_{2}\right|^{2} e_{2}\left(i \lambda_{2}\right)-|z|^{2} e_{2}(i \lambda)+\bar{z}_{1} z_{2} e_{1}\left(i \lambda_{1}\right) e_{1}\left(i \lambda_{2}\right) \\
& +\left|z_{1}\right|^{2} e_{2}\left(-i \lambda_{1}\right)+\left|z_{2}\right|^{2} e_{2}\left(-i \lambda_{2}\right)-|z|^{2} e_{2}(-i \lambda) \\
& +\overline{z_{1} z_{2} e_{1}\left(i \lambda_{1}\right) e_{1}\left(i \lambda_{2}\right)} \\
= & \left|z_{1}\right|^{2}\left(e_{2}\left(i \lambda_{1}\right)+e_{2}\left(-i \lambda_{1}\right)\right)+\left|z_{2}\right|^{2}\left(e_{2}\left(i \lambda_{2}\right)+e_{2}\left(-i \lambda_{2}\right)\right) \\
& -|z|^{2}\left(e_{2}(i \lambda)+e_{2}(-i \lambda)\right)+2 \Re \mathfrak{R e}\left(\bar{z}_{1} z_{2} e_{1}\left(i \lambda_{1}\right) e_{1}\left(i \lambda_{2}\right)\right) \\
= & \left|z_{1}\right|^{2}\left(e_{1}\left(i \lambda_{1}\right) e_{1}\left(-i \lambda_{1}\right)\right)+\left|z_{2}\right|^{2}\left(e_{1}\left(i \lambda_{2}\right) e_{1}\left(-i \lambda_{2}\right)\right) \\
& -|z|^{2}\left(e_{1}(i \lambda) e_{1}(-i \lambda)\right)+2 \Re \mathfrak{R e}\left(\bar{z}_{1} z_{2} e_{1}\left(-i \lambda_{1}\right) \mathrm{e}^{i \lambda_{1}} e_{1}\left(i \lambda_{2}\right)\right) \\
= & \left(\left|z_{1} e_{1}\left(i \lambda_{1}\right)\right|^{2}+\left|z_{2} \mathrm{e}^{i \lambda_{1}} e_{1}\left(i \lambda_{2}\right)\right|^{2}+2 \Re \mathfrak{R e}\left(\overline{z_{1} e_{1}\left(i \lambda_{1}\right)} z_{2} \mathrm{e}^{i \lambda_{1}} e_{1}\left(i \lambda_{2}\right)\right)\right) \\
& -\left|z e_{1}(i \lambda)\right|^{2} \\
= & \left|z_{1} e_{1}\left(i \lambda_{1}\right)+z_{2} \mathrm{e}^{i \lambda_{1}} e_{1}\left(i \lambda_{2}\right)\right|^{2}-\left|z_{1}(i \lambda)\right|^{2} \\
= & \left|z e_{1}(i \lambda)\right|^{2}-\left|z e_{1}(i \lambda)\right|^{2} \\
= & 0 .
\end{aligned}
$$


This ends the proof.

In the following, we identify $\mathbb{C}$ to $\mathbb{R}$, via relation

$z=x+i y \equiv(x, y)$.

Then we obtain

$\mathbb{C} \times\left[-\pi, \pi\left[\times \mathbb{R} \equiv \mathbb{R}^{2} \times[-\pi, \pi[\times \mathbb{R}\right.\right.$

and it is clear that $\mathfrak{G}:=\mathbb{R}^{2} \times[-\pi, \pi[\times \mathbb{R}$ is a manifold with boundary.

Theorem 3 The manifold $\mathfrak{G}$ is a Lie group, its law $\circ$ is defined by

$\left(x_{1}, y_{1}, \lambda_{1}, \alpha_{1}\right) \circ\left(x_{2}, y_{2}, \lambda_{2}, \alpha_{2}\right)=(x, y, \lambda, \alpha)$,

where $(x, y, \lambda, \alpha)$ is given as follows:

Putting $z_{j}=x_{j}+i y_{j}, j=1,2$, then

$\lambda=\lambda_{1}+\lambda_{2} \bmod (2 \pi \mathbb{Z})$,

$x=\mathfrak{R e}\left(\frac{e_{1}\left(i \lambda_{1}\right) z_{1}+\mathrm{e}^{i \lambda_{1}} e_{1}\left(i \lambda_{2}\right) z_{2}}{e_{1}(i \lambda)}\right)$,

$y=\mathfrak{I m}\left(\frac{e_{1}\left(i \lambda_{1}\right) z_{1}+\mathrm{e}^{i \lambda_{1}} e_{1}\left(i \lambda_{2}\right) z_{2}}{e_{1}(i \lambda)}\right)$,

$\alpha=\alpha_{1}+\alpha_{2}+i\left(e_{2}\left(i \lambda_{1}\right)\left|z_{1}\right|^{2}+e_{2}\left(i \lambda_{2}\right)\left|z_{2}\right|^{2}-e_{2}(i \lambda)\left(|x|^{2}+|y|^{2}\right)\right.$

$\left.+e_{1}\left(i \lambda_{1}\right) e_{1}\left(i \lambda_{2}\right) \overline{z_{1}} z_{2}\right)$.

Definition 4 The Lie group $\mathfrak{G}$ is called the (one-mode) generalized Heisenberg group, we denote it by GHeis(1). The domain $\mathfrak{D} \equiv \mathbb{R}^{2} \times\left[-\pi, \pi\left[^{2}\right.\right.$ is called the principal domain of the generalized Heisenberg group.

Proposition 3 The map

$\mathfrak{G} \longrightarrow \Gamma(\mathbb{C}), \quad(x, y, \lambda, \alpha) \longmapsto W(x+i y, \lambda, \alpha)$

is a unitary representation of the generalized Heisenberg group over the Fock space $\Gamma(\mathbb{C})$.

Remark 4 The one-mode Heisenberg group Heis(1) is a subgroup of the generalized Heisenberg group.

Fact:

$\mathbb{R}^{3} \equiv \mathbb{R}^{2} \times\{0\} \times \mathbb{R} \subset \mathbb{R}^{2} \times[-\pi, \pi[\times \mathbb{R}=\mathfrak{G}$.

According to the law $\circ$ above, we have:

If $\lambda_{1}=\lambda_{2}=0$, then from (76), $\lambda=0$ and using (77) and (78), we obtain

$x=\mathfrak{R e}\left(z_{1}+z_{2}\right)=x_{1}+x_{2} ; \quad y=\mathfrak{I m}\left(z_{1}+z_{2}\right)=y_{1}+y_{2}$.

Using (79), one has

$$
\begin{aligned}
\alpha & =\alpha_{1}+\alpha_{2}+i\left(\frac{1}{2}\left|z_{1}\right|^{2}+\frac{1}{2}\left|z_{2}\right|^{2}-\frac{1}{2}\left|z_{1}+z_{2}\right|^{2}+\overline{z_{1}} z_{2}\right) \\
& =\alpha_{1}+\alpha_{2}+i\left(-\mathfrak{R e}\left(\overline{z_{1}} z_{2}\right)+\overline{z_{1}} z_{2}\right) \\
& =\alpha_{1}+\alpha_{2}-\mathfrak{I m}\left(\overline{z_{1}} z_{2}\right) \\
& =\alpha_{1}+\alpha_{2}+\mathfrak{I m}\left(z_{1} \overline{z_{2}}\right) \\
& =\alpha_{1}+\alpha_{2}+y_{1} x_{2}-x_{1} y_{2} .
\end{aligned}
$$

Then

$\left(x_{1}, y_{1}, 0, \alpha_{1}\right) \circ\left(x_{2}, y_{2}, 0, \alpha_{2}\right)=\left(x_{1}+x_{2}, y_{1}+y_{2}, 0, \alpha_{1}+\alpha_{2}+y_{1} x_{2}-x_{1} y_{2}\right)$,

or equivalently

$\left(x_{1}, y_{1}, \alpha_{1}\right) *\left(x_{2}, y_{2}, \alpha_{2}\right)=\left(x_{1}+x_{2}, y_{1}+y_{2}, \alpha_{1}+\alpha_{2}+y_{1} x_{2}-x_{1} y_{2}\right)$,

which is the usual law of the one-mode Heisenberg group given as in (8). 


\section{References}

1. Accardi, L., Dahri, A.: $C^{*}$-non linear second quantization (2014). arXiv:1401.5500v2

2. Accardi, L., Ouerdiane, H., Rebei, H.: On the quadratic Heisenberg group. Infin. Dimens. Anal. Quantum Probab. Relat. Top. 13(4), 551-587 (2010)

3. Accardi, L., Ouerdiane, H., Rebei, H.: Renormalized square of white noise quantum time shift. Commun. Stoch. Anal. 6(2), 177-191 (2012)

4. Reed, M., Simon, B.: Methods of Mathematical Physics, vol. I. Academic Press, INC. (London) LTD., London 